\title{
Synchronization and intermittency in three-coupled chaotic oscillators
}

$\operatorname{AUTHOR}(\mathrm{S})$ :

Tsukamoto, N; Miyazaki, S; Fujisaka, H

CITATION:

Tsukamoto, N ... [et al]. Synchronization and intermittency in threecoupled chaotic oscillators. Physical Review E 2003, 67(1): 016212.

ISSUE DATE:

2003-01

URL:

http://hdl.handle.net/2433/50285

RIGHT:

Copyright 2003 American Physical Society 
PHYSICAL REVIEW E 67, 016212 (2003)

\title{
Synchronization and intermittency in three-coupled chaotic oscillators
}

\author{
Nobunari Tsukamoto, ${ }^{*}$ Syuji Miyazaki, ${ }^{\dagger}$ and Hirokazu Fujisaka ${ }^{\ddagger}$ \\ Department of Applied Analysis and Complex Dynamical Systems, Graduate School of Informatics, Kyoto University, \\ Kyoto 606-8501, Japan
}

(Received 9 July 2002; published 28 January 2003)

\begin{abstract}
Synchronization of three-coupled chaotic oscillators was studied with the use of a coupled map system derived for interacting kicked relaxators. Partial synchronization (PS), in which two of the three were synchronized, was observed in addition to complete synchronization. An intermittency associated with the breakdown of the PS, seemingly different from the conventional on-off intermittency, was found. We elucidated the statistics, observing the burst-size distribution, the laminar duration distribution, etc. It was found that the breakdown of the PS generated an anomalous diffusion different from that associated with on-off intermittency.

DOI: 10.1103/PhysRevE.67.016212

PACS number(s): 05.45.Ac, 05.45.Xt, 05.40.Fb
\end{abstract}

\section{INTRODUCTION}

Synchronization and desynchronization are quite ubiquitous problems in coupled dynamical systems composed of limit cycles or even chaotic oscillators [1,2]. Synchronization among coupled periodic oscillators was first recognized by Huygens. On the other hand, even in coupled chaotic oscillators, synchronization is observed when the trajectory instability present in a chaos oscillator element is suppressed by the coupling between oscillators. The synchronization between coupled chaotic oscillators was reported in Ref. [3].

When chaos synchronization is interrupted due to a change in coupling strength, an intermittency occurs, which is different from those associated with either the destruction or the instability of limit cycle oscillation, known as the Pomeau-Manneville intermittency [4]. This is called the modulational intermittency or the on-off intermittency. Chaos synchronization and the on-off intermittency are observed not only in mathematical models [5-16], but also in condensed matter physics and engineering [17-25], and are typical nonlinear phenomena associated with coupled chaotic oscillator systems.

The chaos synchronization attracts attention not only from the viewpoint of ubiquity in nonlinear dynamics but also from the applicability to engineering. In fact, after Pecora and Caroll [26] showed that the chaos synchronization is applicable to secret communication, numerous attempts have been proposed to apply chaos synchronization to engineering. Furthermore, in addition to complete synchronization, several kinds of synchronization such as partial synchronization and phase synchronization have recently been proposed in many different contexts of coupled oscillator systems [1].

The present study examined the synchronization and the desynchronization problems in a coupled three chaotic oscillator system to clarify their differences from those in a coupled two-oscillator system. We discuss the synchronization-desynchronization phenomenon with as

\footnotetext{
*Electronic address: tsukamo@acs.i.kyoto-u.ac.jp

${ }^{\dagger}$ Corresponding author. Electronic address: syuji@acs.i.kyotou.ac.jp

ॠElectronic address: fujisaka@acs.i.kyoto-u.ac.jp
}

simple a model as possible. In order to do this, in Sec. II, we construct a coupled map system using interacting kicked relaxators for a general network system. In Sec. III, we examine the complete and partial synchronization of an equivalently coupled three-chaotic map system, calculating the stability parameters. The statistical dynamics associated with the breakdown of the partial synchronization are studied by paying particular attention to comparison with the statistics of on-off intermittency. In Sec. IV, we discuss two kinds of diffusion caused by the breakdown of partial synchronization. Based on the continuous-time random walk (CTRW) theory, we analytically derive mean square displacements (MSDs) to find anomalous subdiffusion and ballistic motion followed by normal diffusion. The statistical properties in various scaling forms are also derived. We compare the theory with numerical results in this section. Section V is devoted to concluding remarks. The general framework of the CTRW and its implementation in our model are described in the Appendix.

\section{MODEL OF THE COUPLED OSCILLATOR SYSTEM}

Let us consider the $N$-coupled kicked relaxator system given by

$$
\begin{gathered}
\frac{d x^{(j)}(t)}{d t}=p^{(j)}(t), \\
\epsilon \frac{d p^{(j)}(t)}{d t}=-p^{(j)}+F\left(x^{(j)}(t)\right) \sum_{n=-\infty}^{\infty} \delta\left(t-t_{n}\right)+\mathcal{D} x^{(j)}(t),
\end{gathered}
$$

where $x^{(j)}$ and $p^{(j)}$ are, respectively, the physical quantity and the corresponding momentum of the $j$ th relaxator, $t_{n}$ $=n T(n=0,1,2,3, \ldots)$,$T being the period of kicks, are the$ times when the kicks are given and $\epsilon(>0)$ is a smallness parameter. We impose the cyclic boundary condition $x^{(N+1)}$ $=x^{(1)}$ and $p^{(N+1)}=p^{(1)}$. The term $\mathcal{D} x^{(j)}$ denotes a linear coupling term among relaxators, and, e.g., has

$$
\mathcal{D} x^{(j)}=C\left(x^{(j-1)}-2 x^{(j)}+x^{(j+1)}\right)
$$


for the spring-type coupling for an array of oscillators. For a moment, however, we do not require an explicit form of coupling.

By putting

$$
M=\left(\begin{array}{cc}
0 & 1 \\
\epsilon^{-1} \mathcal{D} & -\epsilon^{-1}
\end{array}\right),
$$

the integration of Eqs. (1) and (2) for $t_{n}-\delta \leqslant t \leqslant t_{n+1}-\delta, \delta$ being a positive infinitesimal quantity, gives

$$
\left(\begin{array}{c}
x_{n+1}^{(j)} \\
p_{n+1}^{(j)}
\end{array}\right)=\sum_{\ell=1}^{N} \phi_{j \ell}\left(\begin{array}{c}
x_{n}^{(\ell)} \\
p_{n}^{(\ell)}+\epsilon^{-1} F\left(x_{n}^{(\ell)}\right)
\end{array}\right),
$$

where we put $x_{n}^{(j)}=x^{(j)}\left(t_{n}-\delta\right)=x^{(j)}\left(t_{n}\right)$ and $p_{n}^{(j)}=p^{(j)}\left(t_{n}\right.$ $-\delta)$. Here, $\phi_{j \ell}$ is the matrix defined by

$$
e^{T M}\left(\begin{array}{c}
\xi^{(j)} \\
\eta^{(j)}
\end{array}\right)=\sum_{\ell=1}^{N} \phi_{j \ell}\left(\begin{array}{c}
\xi^{(\ell)} \\
\eta^{(\ell)}
\end{array}\right) .
$$

We have used the fact that $x^{(j)}(t)$ is continuous at $t=t_{n}$, while $p^{(j)}(t)$ is discontinuous at $t=t_{n}$.

Let us take the limit $\epsilon \rightarrow 0$. After a short manipulation, the evaluation of Eq. (5) for $\epsilon \rightarrow 0$ leads to

$$
x_{n+1}^{(j)}=\sum_{\ell=1}^{N} J_{j \ell} f\left(x_{n}^{(\ell)}\right),
$$

and $p_{n+1}=\mathcal{D} x_{n+1}^{(j)}$, where

$$
f(x)=x+F(x)
$$

is the local map. The coupling kernel $J_{j \ell}$ is defined by

$$
e^{T \mathcal{D}} g_{j}=\sum_{\ell=1}^{N} J_{j \ell} g_{\ell},
$$

where $g_{j}$ is an arbitrary function of the lattice point $j$. Equation (7) is an extension of the derivation of the mapping system for a single relaxator with kicks and is the fundamental mapping system studied later in this paper. It is worthwhile to note that for the present type of coupling $\Sigma_{j} \mathcal{D} x^{(j)}$ $=0$, one gets

$$
\sum_{j=1}^{N} J_{j \ell}=\sum_{\ell=1}^{N} J_{j \ell}=1
$$

This is the crucial condition for the existence of complete synchronized oscillation $x_{n}^{(1)}=x_{n}^{(2)}=\cdots=x_{n}^{(N)} \equiv x_{n}^{0}$, which obeys $x_{n+1}^{0}=f\left(x_{n}^{0}\right)$.

As simple models, let us consider the cases with $N$ $=2,3$, and 4 with the coupling type (3). For $N=2$, one finds

$$
\begin{gathered}
x_{n+1}^{(j)}=f\left(x_{n}^{(j)}\right)+J\left[f\left(x_{n}^{(\ell)}\right)-f\left(x_{n}^{(j)}\right)\right], \\
J=\frac{1}{2}\left(1-e^{-2 C T}\right),
\end{gathered}
$$

where $(j, \ell)=(1,2),(2,1)$. For $N=3$, one easily obtains

$$
\begin{gathered}
x_{n+1}^{(j)}=f\left(x_{n}^{(j)}\right)+J\left[f\left(x_{n}^{(j-1)}\right)-2 f\left(x_{n}^{(j)}\right)+f\left(x_{n}^{(j+1)}\right)\right] \\
(j=1,2,3), \\
J=\frac{1}{3}\left(1-e^{-3 C T}\right),
\end{gathered}
$$

where $x_{n}^{(0)}=x_{n}^{(4)} \equiv x_{n}^{(3)}$. Furthermore, for $N=4$, the coupled map system is given by

$$
\begin{aligned}
x_{n+1}^{(j)}= & f\left(x_{n}^{(j)}\right)+J_{1}\left[f\left(x_{n}^{(j-1)}\right)-2 f\left(x_{n}^{(j)}\right)+f\left(x_{n}^{(j+1)}\right)\right] \\
& +J_{2}\left[f\left(x_{n}^{(j-2)}\right)-2 f\left(x_{n}^{(j)}\right)+f\left(x_{n}^{(j+2)}\right)\right],
\end{aligned}
$$

$$
J_{1}=\frac{1}{4}\left(1-e^{-4 C T}\right), \quad J_{2}=\frac{1}{8}\left(1-2 e^{-2 C T}+e^{-4 C T}\right),
$$

where $x_{n}^{(-1)}=x_{n}^{(3)}, x_{n}^{(0)}=x_{n}^{(4)}, x_{n}^{(5)}=x_{n}^{(1)}$, and $x_{n}^{(6)}=x_{n}^{(2)}$. In the present paper, using the the three-coupled map system (13), we will study the synchronization-desynchronization phenomenon, paying particular attention to statistics near the breakdown of partial synchronization.

\section{SYNCHRONIZATION AND ITS BREAKDOWN IN THE COUPLED THREE-CHAOTIC MAP SYSTEM}

\section{A. Two types of synchronization and their linear stability}

The three-coupled map model used in this paper is the symmetrically coupled system,

$$
\begin{aligned}
& x_{n+1}^{(1)}=f\left(x_{n}^{(1)}\right)+J\left[f\left(x_{n}^{(2)}\right)-2 f\left(x_{n}^{(1)}\right)+f\left(x_{n}^{(3)}\right)\right], \\
& x_{n+1}^{(2)}=f\left(x_{n}^{(2)}\right)+J\left[f\left(x_{n}^{(3)}\right)-2 f\left(x_{n}^{(2)}\right)+f\left(x_{n}^{(1)}\right)\right], \\
& x_{n+1}^{(3)}=f\left(x_{n}^{(3)}\right)+J\left[f\left(x_{n}^{(1)}\right)-2 f\left(x_{n}^{(3)}\right)+f\left(x_{n}^{(2)}\right)\right] .
\end{aligned}
$$

Total and partial synchronization, bifurcations, and riddling of the basin structure of a similar system are also analyzed in Refs. [2,27]. Hereafter, we will use the logistic map $f(x)$ $=\operatorname{ax}(1-x)$ with $a=3.8$ whose local dynamics $x_{n+1}$ $=f\left(x_{n}\right)$ has the Lyapunov exponent $\lambda=0.43212 \ldots$. The coupling constant is given as $J=\left(1-e^{-D}\right) / 3$, where $D(>0)$ is chosen as the control parameter.

It is easily shown that Eq. (17) has two types of particular motions. One is the completely synchronized motion (CS),

$$
x_{n}^{(1)}=x_{n}^{(2)}=x_{n}^{(3)} \equiv X_{n}^{(0)},
$$

and the other is the partial synchronized motion (PS),

$$
\begin{gathered}
x_{n}^{(i)}=x_{n}^{(j)} \equiv X_{n}^{(1)}, \\
X_{n}^{(2)} \equiv x_{n}^{(k)} \neq X_{n}^{(1)} \quad(i \neq j \neq k) .
\end{gathered}
$$

In the $\mathrm{CS}$, the coupled oscillator system looks like a single oscillator obeying $X_{n+1}^{(0)}=f\left(X_{n}^{(0)}\right)$. On the other hand, in the PS the time evolution is determined by two degrees of free$\operatorname{dom} X_{n}^{(1)}$ and $X_{n}^{(2)}$. Although these particular motions exist, their observability depends on their stability. 
The linear stability of these solutions is determined by the transverse Lyapunov exponent corresponding to each particular motion. First, we will discuss the stability of the completely synchronized solution $X_{n}^{(0)}$. In the CS, we find

$$
X_{n+1}^{(0)}=f\left(X_{n}^{(0)}\right) .
$$

Three oscillators evolve in time just like one oscillator. By adding infinitesimal perturbations $\delta_{n}^{(i)}$ around the particular motion (18),

$$
x_{n}^{(i)}=X_{n}^{(0)}+\delta_{n}^{(i)} \quad(i=1,2,3),
$$

we get the perturbation equation

$$
\left(\begin{array}{c}
\delta_{n+1}^{(1)} \\
\delta_{n+1}^{(2)} \\
\delta_{n+1}^{(3)}
\end{array}\right)=f^{\prime}\left(X_{n}^{(0)}\right)\left(\begin{array}{ccc}
1-2 J & J & J \\
J & 1-2 J & J \\
J & J & 1-2 J
\end{array}\right)\left(\begin{array}{c}
\delta_{n}^{(1)} \\
\delta_{n}^{(2)} \\
\delta_{n}^{(3)}
\end{array}\right),
$$

where $f^{\prime}(X)=d f(X) / d X$. The magnitude of the perturbation $\bar{\delta}_{n}=\left|\delta_{n}^{(1)}+\delta_{n}^{(2)}+\delta_{n}^{(3)}\right| / 3 \quad$ obeys $\quad \bar{\delta}_{n+1}=\left|f^{\prime}\left(X_{n}^{(0)}\right)\right| \bar{\delta}_{n}$ $=e^{\ln \left|f^{\prime}\left(X_{n}^{(0)}\right)\right|} \bar{\delta}_{n}$. On the other hand, the magnitude of the relative difference $r_{n} \equiv\left|\delta_{n}^{(i)}-\delta_{n}^{(j)}\right| / 2(i \neq j)$ obeys

$$
r_{n+1}=\left|f^{\prime}\left(X_{n}^{(0)}\right)\right| e^{-D} r_{n}=e^{\Lambda_{n}^{(0)}} r_{n},
$$

where $\Lambda_{n}^{(0)}=\ln \left|f^{\prime}\left(X_{n}^{(0)}\right)\right|-D$ is called the local transverse expansion rate. There are three identical equations for three choices of the oscillator numbers $i$ and $j$. In terms of the quantity

$$
\lambda_{\|}=\left\langle\ln \left|f^{\prime}\left(X_{n}^{(0)}\right)\right|\right\rangle=\lim _{N \rightarrow \infty} \frac{1}{N} \sum_{j=1}^{N} \ln \left|f^{\prime}\left(X_{j}^{(0)}\right)\right|,
$$

which is identical to the Lyapunov exponent of the local dynamics $x_{n+1}=f\left(x_{n}\right)$, i.e., $\lambda_{\|}=\lambda=0.43212 \ldots$, we find $\bar{\delta}_{n} \sim \bar{\delta}_{0} e^{\lambda \|^{n}}$. Namely, $\bar{\delta}_{n}$ grows exponentially in time. This is a result of the chaotic local dynamics. This fact is irrelevant to the stability of the CS. The linear stability of the CS is, on the other hand, determined by the temporal average $\lambda_{\perp}^{(0)}$ of $\Lambda_{\perp}^{(0)}$, which is called the transverse Lyapunov exponent, given by

$$
\lambda_{\perp}^{(0)}=\left\langle\Lambda_{n}^{(0)}\right\rangle=\lambda_{\|}-D
$$

If $\lambda_{\perp}^{(0)}<0$, the completely synchronized motion $X_{n}^{(0)}$ is linearly stable against a small deviation from the complete synchronization. Therefore, when $D>\lambda_{\|}=0.43212 \ldots \equiv D_{c}^{0}$, the completely synchronized motion is stable. On the other hand, for $\lambda_{\perp}^{(0)}>0$, i.e., $D<D_{c}^{0}$, the CS is linearly unstable. This means that as $D$ is gradually decreased from above $D_{c}^{0}$, the CS breaks down at $D_{c}^{0}$.

Next, we consider the linear stability of the partially synchronized motion. By putting $x_{n}^{(1)}=x_{n}^{(3)} \equiv X_{n}^{(1)}$, and substituting Eq. (19) into Eq. (17), the state variables in the PS obey the equations of motion,

$$
\begin{gathered}
X_{n+1}^{(1)}=f\left(X_{n}^{(1)}\right)+J\left[f\left(X_{n}^{(2)}\right)-f\left(X_{n}^{(1)}\right)\right], \\
X_{n+1}^{(2)}=f\left(X_{n}^{(2)}\right)+2 J\left[f\left(X_{n}^{(1)}\right)-f\left(X_{n}^{(2)}\right)\right] .
\end{gathered}
$$

This set of equations of motion is composed of two types of oscillators similar to Eq. (11). The crucial difference is however that the coupling is symmetric in Eq. (11) but it is asymmetric in the present case. The linear stability of the PS can be examined by adding a small perturbation from the PS as

$$
\begin{gathered}
x_{n}^{(i)}=X_{n}^{(1)}+\delta_{n}^{(i)} \quad(i=1,3), \\
x_{n}^{(2)}=X_{n}^{(2)}+\delta_{n}^{(2)} .
\end{gathered}
$$

The perturbed equations are given by

$$
\left(\begin{array}{c}
\delta_{n+1}^{(1)} \\
\delta_{n+1}^{(2)} \\
\delta_{n+1}^{(3)}
\end{array}\right)=\left(\begin{array}{ccc}
f^{\prime}\left(X_{n}^{(1)}\right)(1-2 J) & f^{\prime}\left(X_{n}^{(2)}\right) J & f^{\prime}\left(X_{n}^{(1)}\right) J \\
f^{\prime}\left(X_{n}^{(1)}\right) J & f^{\prime}\left(X_{n}^{(2)}\right)(1-2 J) & f^{\prime}\left(X_{n}^{(1)}\right) J \\
f^{\prime}\left(X_{n}^{(1)}\right) J & f^{\prime}\left(X_{n}^{(2)}\right) J & f^{\prime}\left(X_{n}^{(1)}\right)(1-2 J)
\end{array}\right)\left(\begin{array}{c}
\delta_{n}^{(1)} \\
\delta_{n}^{(2)} \\
\delta_{n}^{(3)}
\end{array}\right) .
$$

The magnitude of the deviation from the PS is measured by $r_{n} \equiv\left|\delta_{n}^{(1)}-\delta_{n}^{(3)}\right|$. From Eq. (28), we get

$$
r_{n+1}=\left|f^{\prime}\left(X_{n}^{(1)}\right)\right| e^{-D} r_{n} \equiv e^{\Lambda_{n}^{(1)}} r_{n},
$$

where we defined the transverse Lyapunov exponent $\Lambda_{n}^{(1)}$ for the PS by $\Lambda_{n}^{(1)}=\ln \left|f^{\prime}\left(X_{n}^{(1)}\right)\right|-D$. The linear stability of the PS is determined by its time average

$$
\lambda_{\perp}^{(1)}=\left\langle\Lambda_{n}^{(1)}\right\rangle=\lambda^{(1)}-D,
$$

where

$$
\lambda^{(1)}=\left\langle\ln \left|f^{\prime}\left(X_{n}^{(1)}\right)\right|\right\rangle=\lim _{N \rightarrow \infty} \frac{1}{N} \sum_{j=1}^{N} \ln \left|f^{\prime}\left(X_{j}^{(1)}\right)\right| .
$$

This formula looks the same as the transverse Lyapunov exponent (25) for the completely synchronized solution. However, in contrast to that, $\lambda_{\|}$is independent of the coupling constant and has the meaning of the largest Lyapunov exponent of the CS, $\lambda^{(1)}$ depends on the coupling constant $D$ and is different from the largest Lyapunov exponent of the PS. 


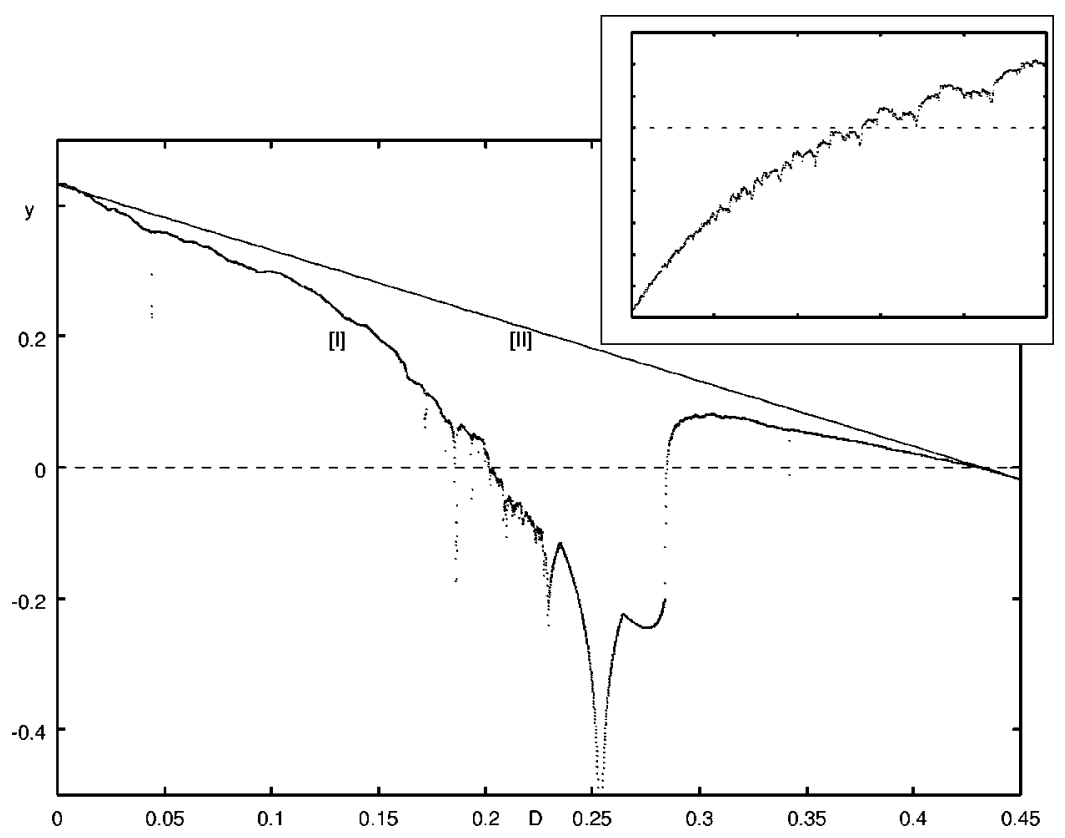

FIG. 1. Coupling constant $(D)$ dependence on the transverse Lyapunov exponents $(y) \lambda_{\perp}^{(0)}[\mathrm{II}]$ or $\lambda_{\perp}^{(1)}[\mathrm{I}]$ relevant to the stability of the completely synchronized motion and the partially synchronized motion, respectively. The inset is a blowup $(0.284 \leqslant D \leqslant 0.285$ and $-0.06 \leqslant y \leqslant 0.03)$.

Figure 1 shows the numerical results of the two kinds of the transverse Lyapunov exponents plotted as function of the coupling constant $D . \lambda_{\perp}^{(0)}$ is just the formula (25). On the other hand, $\lambda_{\perp}^{(1)}$ in Fig. 1 was calculated as follows. For an initial condition $X_{0}^{(1)}, X_{0}^{(2)}\left(\neq X_{0}^{(1)}\right)$, we solved Eq. (26) for a given $D$. If the state variables eventually approach $X_{n}^{(1)}$ $=X_{n}^{(2)}$ for almost arbitrary initial conditions, the CS is stable for that value of $D$. When the state variables do not approach the CS motion, the dynamics are identical to the PS, and then we calculated $\lambda_{\perp}^{(1)}$, carrying out the time average (31). Figure 1 shows that $\lambda_{\perp}^{(1)}$ sensitively depends on $D$ because of the existence of the window structure. There are three characteristic values of $D$, where $\lambda_{\perp}^{(1)}$ changes its sign as $D$ is changed. They are $D_{0}=0.43212 \ldots, D_{c}^{1} \sim 0.28455$, and $D_{c}^{2} \sim 0.20145$. At $D=D_{c}^{0}$, the stability of the CS changes. On the other hand, for $D$ slightly above $D_{c}^{1}$ and slightly below $D_{c}^{2}$, the partial synchronization breaks down, and the three oscillators evolve in time in a desynchronized way. Near $D_{c}^{2}$ the dynamics sensitively depend on $D$ because of the window structure of the system. Hereafter, we will study the statistical characteristics near $D=D_{c}^{1}$.

In order to observe the synchronization, we define

$$
\Delta_{n}^{i, j} \equiv x_{n}^{(i)}-x_{n}^{(j)}
$$

If $\Delta_{n}^{i, j}$ vanishes, then the $i$ th and $j$ th oscillators are synchronized. The bifurcation diagrams of the system for a change of $D$ are displayed in Fig. 2. Figure 2(a) shows the bifurcation diagram obtained by gradually increasing $D$ starting at $D=0$, and Fig. 2(b) is the result by gradually decreasing $D$ starting at $D=0.45\left(>D_{c}^{0}\right)$. Comparing Figs. 1 and 2, we find that complete synchronization for $\lambda_{\perp}^{(0)}<0$ and partial synchronization for $D$ with $\lambda_{\perp}^{(0)}>0, \lambda_{\perp}^{(1)}<0$ are observed. Furthermore, for $D_{c}^{2}<D<0.2445$, two kinds of dynamical be- havior are observed; the bifurcation diagrams thus show hysteresis, which implies the coexistence of several attractors in this range of $D$.

\section{B. Statistics near $D_{c}^{1}$}

First, consider $D<D_{c}^{1}$, where $\lambda_{\perp}^{(0)}>0, \lambda_{\perp}^{(1)}<0$, and therefore complete synchronization is unstable, while the partial synchronization is stable (Fig. 3). Slightly below $D_{c}^{1}$, the system eventually falls into the partial synchronization state in which two of three oscillators are synchronized for almost all initial conditions, showing a chaotic motion. Once the system falls in this partial synchronization state, the two synchronized oscillators are never separated. Since the pair of oscillators which become synchronized depends on the initial conditions, it is evident that there exist three equivalent partial synchronization states. This chaos shows no strong temporal correlation. The probability density for the state variable difference between desynchronized oscillators is shown in Fig. 4. Apparently it is different from the power law statistics of the on-off intermittency $\left(\sim l^{-\eta}, \eta \approx 1\right)$. As explained above, when the partial synchronization is stable, two oscillators eventually become synchronized. Namely, one of the partial synchronization attractors is completely determined by the initial condition. It has been shown that an attractor is sensitive to the initial conditions and this is possibly another example of the riddled basin recently extensively studied in connection with the generation of on-off intermittency [2,27].

Next, consider the dynamics in the parameter region of $D$ slightly above $D>D_{c}^{1}$, where $\lambda_{\perp}^{(1)}>0$ and the partial synchronized solution is weakly unstable. Figure 5 displays a typical time series of differences of the state variables. Since the partial synchronized state is weakly unstable, the state points remain for a long time near the three former partial synchronization attractors for $D<D_{c}^{1}$, chaotically jumping among them. In this way, the present chaotic time series are 

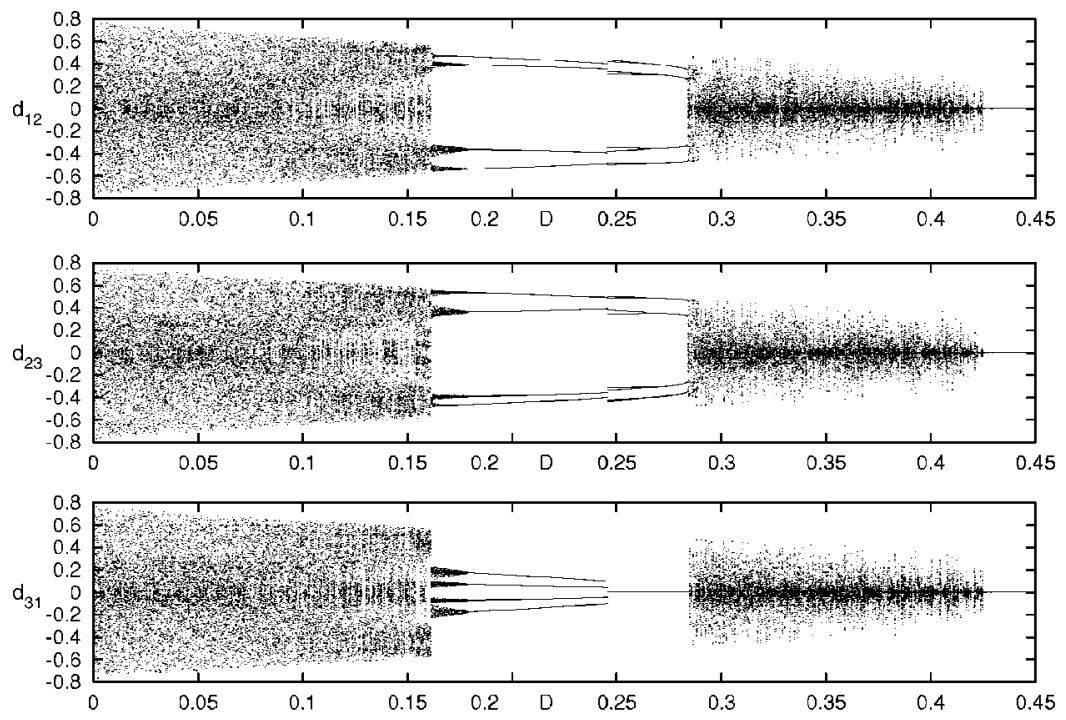

(a)
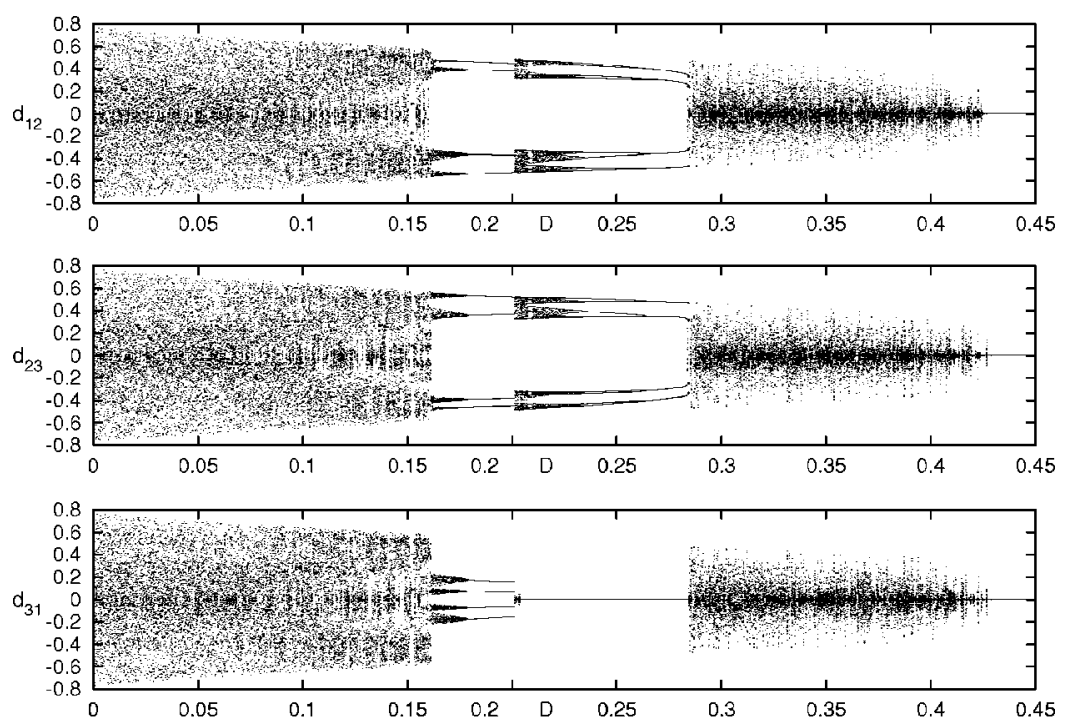

(b)
FIG. 2. Bifurcation diagrams for the threecoupled oscillator system as the coupling constant $D$ is changed, where $d_{i j}=\Delta_{n}^{i, j}=x_{n}^{(i)}-x_{n}^{(j)}$. Figures (a) and (b) are the bifurcation diagrams obtained by, respectively, increasing and decreasing $D$. One observes hysteresis in the intermediate range of the coupling constant. characterized as intermittent switching among the former partial synchronization attractors. This situation is quite analogous to the case in which the complete synchronization is weakly broken as the control parameter is changed and one observes on-off intermittency. In order to study the statistics, we observed the laminar length distribution $\rho(\tau)$, where the laminar length $\tau$ stands for the duration of one of the quasipartial synchronized motions near the former attractors. Figure 6 depicts the numerically obtained laminar length distribution $\rho(\tau)$. It seems that the probability density shows the $\rho(\tau) \sim \tau^{-3 / 2}$ law, which is an eminent characteristic of on-off intermittency [18], particularly in a long duration region $(\tau$ $>10^{3}$ ). In contrast to the on-off intermittency case, a characteristic swelling is present near $\tau \sim 10^{2}$, which may be due to a certain correlation of the present system which is absent in on-off intermittency.

Figure 7 shows the probability density of the amplitude $l\left(=\left|\Delta^{i, j}\right|\right)$. Because of the symmetry of dynamical behaviors of oscillators, the probability density is independent of the choice of the oscillator numbers $i$ and $j(\neq i)$. In the small amplitude region $l<10^{-5}$, the probability density has the power law form $p(l) \sim l^{-a}(a \approx 1)$. This exponent is the same as that observed in the on-off intermittency just after its onset. On the other hand, in the region $l>10^{-3}$, the probability density deviates from the power law and looks identical to that in the asymmetrically coupled chaos showing partial synchronization (Fig. 3).

Figure 8 shows the power spectrum of $\Delta_{n}^{1,2}$, which is again independent of the choice of the oscillator numbers $i$ and $j$. By noting that there are three characteristic regimes in the frequency space, it is expected that there may exist two characteristic time scales in the time series. The characteristics are similar to those observed in on-off intermittency except for the large frequency region.

We then studied the time series of the quantity defined by 

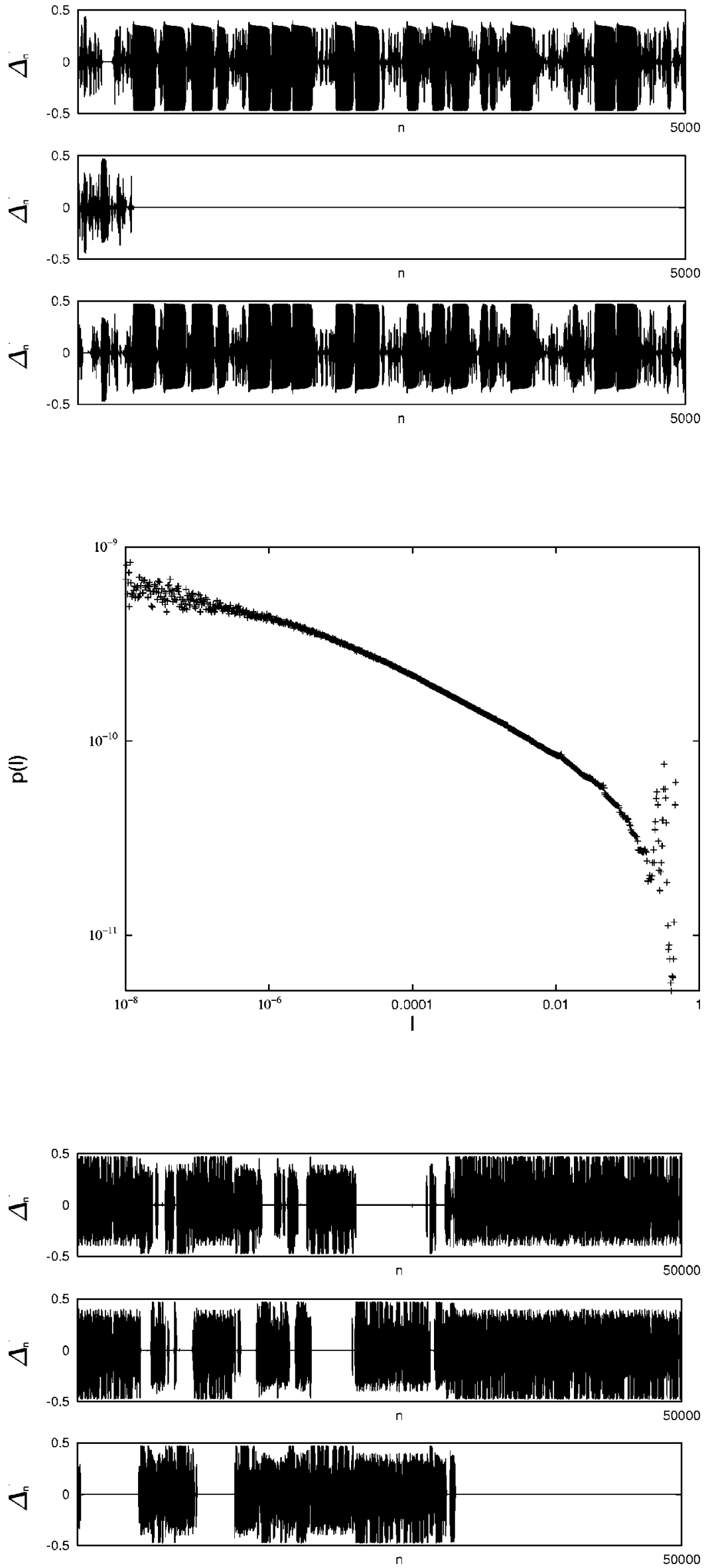

FIG. 3. Temporal evolutions of the differences $\Delta_{n}^{i, j}$ of state variables for an initial condition arbitrarily chosen for the coupling constant $D=0.284\left(<D_{c}^{1}\right)$. One observes a partial synchronization.
FIG. 4. Probability density of the magnitude $l_{n}=\left|\Delta_{n}^{1,2}\right|=\left|\Delta_{n}^{3,1}\right|$ in the partial synchronization state. It is evident that the characteristic is different from that of on-off intermittency.
FIG. 5. Typical time series of differences of state variables in the region where the partial synchronization is weakly unstable, $[D=0.2846$ $\left.\left(>D_{c}^{1}\right)\right]$. One observes an intermittent switching among the three former partial synchronization attractors. 

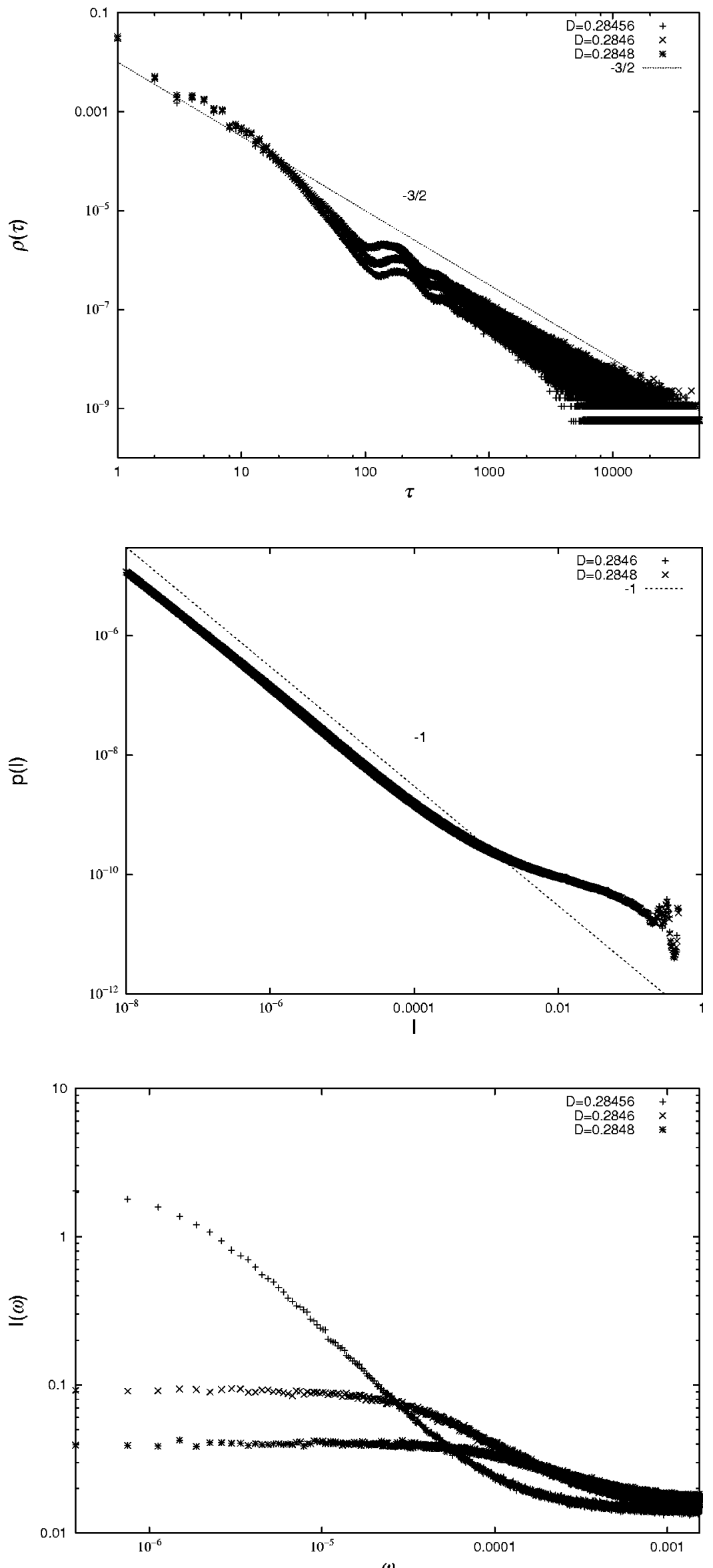

FIG. 7. Probability density of the amplitude $l=\left|\Delta_{n}^{i, j}\right|$ for $D=0.2846,0.2848$ with $D \gtrsim D_{c}^{1}$. The asymptotic law $P(l) \propto l^{-1}$ appears in the small amplitude region.

FIG. 6. Probability density of the duration time of the quasipartial synchronization for $D$ $=0.28456,0.2846,0.2848 .10^{5}-10^{6}$ ensemble members are used for the calculation.

FIG. 8. Power spectra of the time series of the difference $\Delta_{n}^{1,2}$ for $D=0.28456,0.2846,0.2848$. Except for a large frequency region, the characteristic is similar to that of on-off intermittency. 


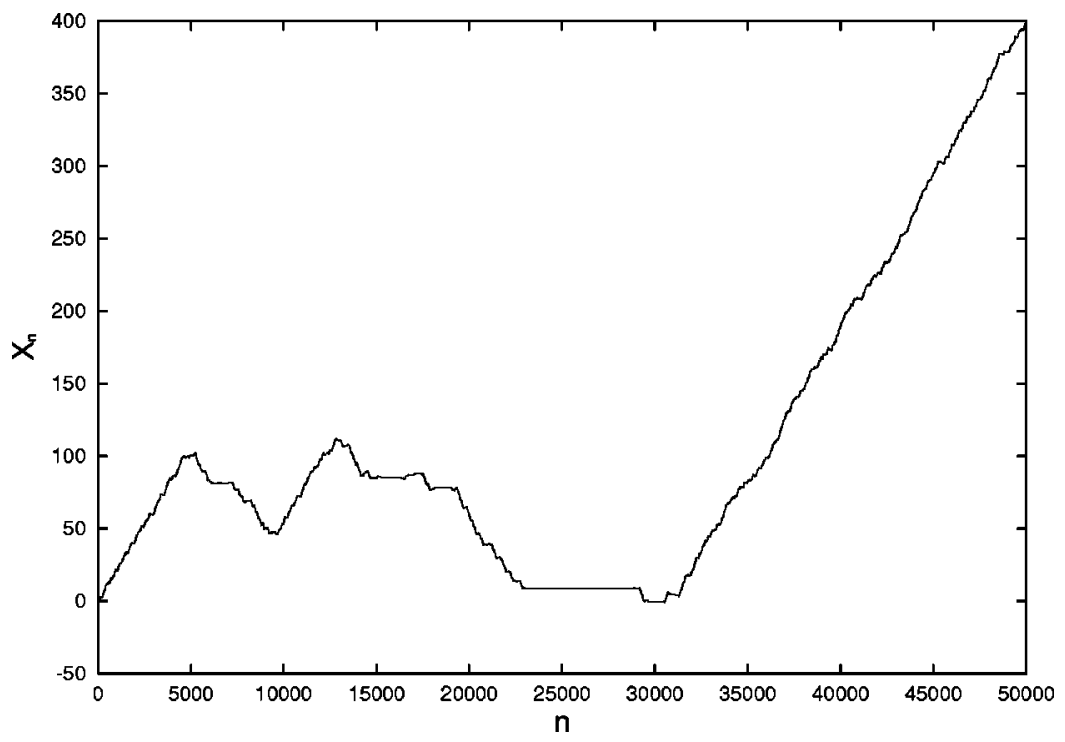

FIG. 9. Time series of $X_{n}$.

$$
\Xi_{n} \equiv \sqrt[3]{\left|\Delta_{n}^{1,2} \Delta_{n}^{2,3} \Delta_{n}^{3,1}\right|}
$$

observing its laminar duration distribution. This quantity measures how the state point is close to the former partial synchronization attractors. Namely, if any of the three $\left|\Delta_{n}^{i, j}\right|$ is small, i.e., if any of the oscillator pairs is close to synchronization, $\Xi_{n}$ takes a small value. The time series of $\Xi_{n}$ is similar to that of on-off intermittency, to yield the probability density of the laminar duration of $\Xi_{n}$ following the $\tau^{-3 / 2}$ law in a large $\tau$ region.

From the above observation, we find that when the partial synchronization is weakly broken the statistics of the dynamics on long-time laminar duration and small amplitude of state variable difference have the same statistics as those of on-off intermittency. One finds from Fig. 5 that in the time region where $\Delta_{n}^{i, j}$ is not sufficiently small, i.e., the $i$ th and $j$ th oscillators are not close to synchronization, the other difference $\Delta_{n}^{j, k}(k \neq i)$ takes a small value, i.e., the $i$ th and the $k(\neq j)$ th oscillators are approximately synchronized. The chaotic evolution in these time regions is almost the same as the (stable) partial synchronized state observed in the asymmetrically coupled equations of motion. In conclusion, the time series of the broken partial synchronization has a structure which puts the laminar phase or the partial synchronization chaos at the laminar phase of on-off intermittency.

\section{TWO KINDS OF DIFFUSION CAUSED BY BREAKDOWN OF PARTIAL SYNCHRONIZATION}

Now, we focus on the statistical properties of breakdown of partial synchronization. As shown in Fig. 5, the phase difference $\Delta_{n}^{1,2}$ consists of the following four states:

(V1) $\Delta_{n}^{1,2} \sim 0$ for $x^{(1)} \sim x^{(2)}$,

(V2) irregular $\Delta_{n}^{1,2}$ with positive average $\left\langle\Delta^{1,2}\right\rangle=v>0$ for $x^{(2)} \sim x^{(3)}$,

(V3) irregular $\Delta_{n}^{1,2}$ with negative average $\left\langle\Delta^{1,2}\right\rangle=-v$ $<0$ for $x^{(3)} \sim x^{(1)}$,

(V4) irregular $\Delta_{n}^{1,2}, \Delta_{n}^{2,3}$, and $\Delta_{n}^{3,1}$ at the same time for no partial synchronization.

The above mentioned (V2) and (V3) are the results from

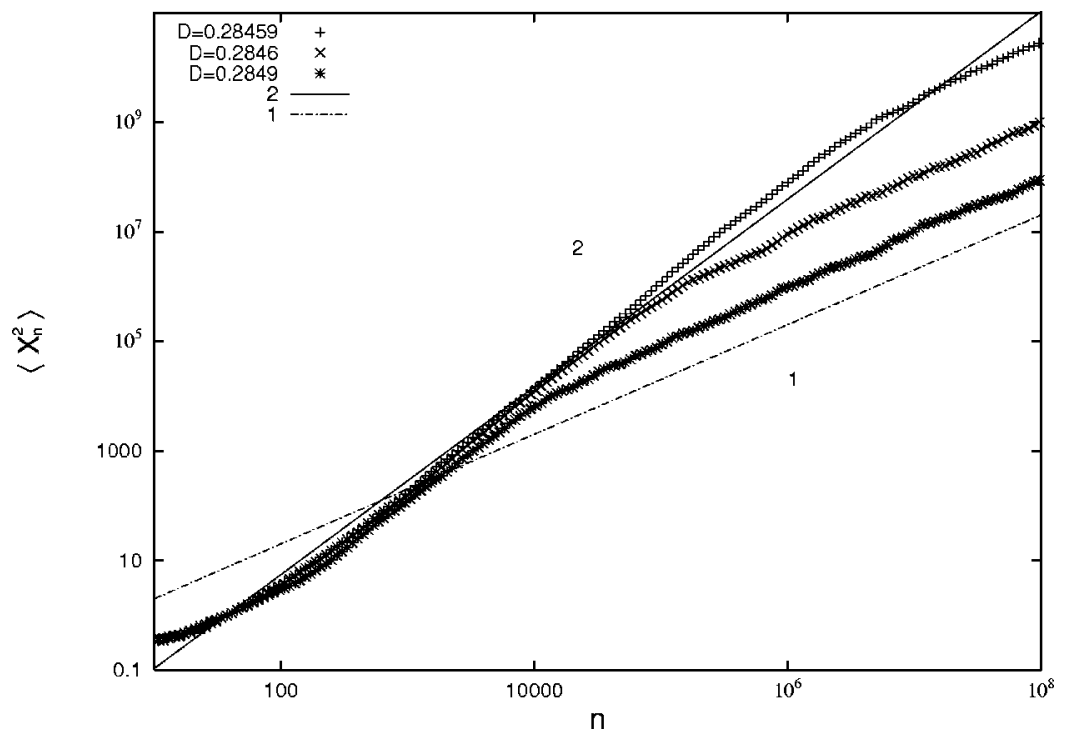

FIG. 10. Mean square displacements $\left\langle X_{n}^{2}\right\rangle$ for $D=0.28459,0.2846,0.2849$. Two kinds of the power laws Eq. (43) are given by lines. 

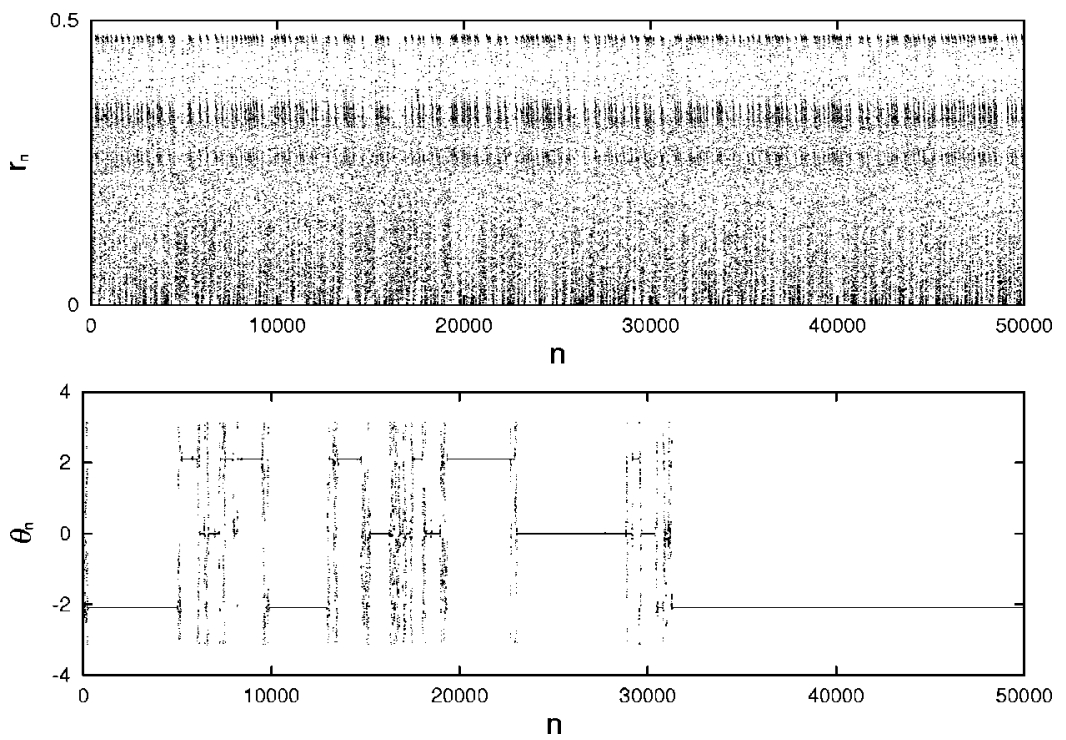

FIG. 11. Time series of $r_{n}$ and $\theta_{n}$. the symmetry of the equation of motion. Let us regard $\Delta_{n}^{1,2}$ as the velocity of a particle, and we define its position at time $n$,

$$
X_{n}=\sum_{t=0}^{n-1} \Delta_{n}^{1,2} .
$$

As the first characterization of statistical properties of the breakdown of partial synchronization, we observe how $X_{n}$ diffuses. The time series of $X_{n}$ is shown in Fig. 9. Corresponding to the above mentioned (V1), (V2), and (V3), quiescent state, almost uniform motion with positive and negative velocity are observed. The element (V4) hardly influences the diffusion at all. The duration time of each of (V1)-(V3) equally distributes according to the power law with the exponent $-3 / 2$ as we mentioned before. MSDs $\left\langle X_{n}^{2}\right\rangle$ are shown in Fig. 10. Note that $\left\langle X_{n}\right\rangle=0$ due to the symmetry. The transition from ballistic motion $\left\langle X_{n}^{2}\right\rangle \propto n^{2}$ to normal diffusion $\left\langle X_{n}^{2}\right\rangle \propto n$ can be observed. We will compare these numerical results with those from the theory by use of CTRW velocity model.

We now look at the switching of the pair of quasipartial synchronizations. In the three-dimensional phase space $\left(x^{(1)}, x^{(2)}, x^{(3)}\right)$, perfect synchronization is described by trajectories on the line $\mathcal{L}, x^{(1)}=x^{(2)}=x^{(3)}$, and partial synchronization is described by trajectories on either of the planes $\mathcal{P}_{1}, x^{(1)}=x^{(2)} ; \mathcal{P}_{2}, x^{(2)}=x^{(3)} ;$ and $\mathcal{P}_{3}, x^{(3)}=x^{(1)}$. We project the phase points onto the plane $\mathcal{P}$ perpendicular to the line $\mathcal{L}$. This line is degenerated to a point, three planes $\mathcal{P}_{i}(i$ $=1,2,3)$ are also degenerated to three lines on the plane $\mathcal{P}$. We define the polar coordinate $\left(r, \theta^{\prime}\right)$ on the plane $\mathcal{P}$ as

$$
\begin{gathered}
r=\sqrt{\frac{\left(x^{(1)}-x^{(2)}\right)^{2}+\left(x^{(2)}-x^{(3)}\right)^{2}+\left(x^{(3)}-x^{(1)}\right)^{2}}{2}}, \\
\cos \theta^{\prime}=\frac{1}{r}\left(\frac{x^{(1)}-x^{(2)}}{2}-x^{(3)}\right),
\end{gathered}
$$

$$
\sin \theta^{\prime}=\frac{\sqrt{3}}{2 r}\left(x^{(1)}-x^{(2)}\right) .
$$

The partial synchronization $x^{(1)}=x^{(2)}$ corresponds to $\theta^{\prime}=0$ and $\theta^{\prime}=\pi$. In order to obtain one-to-one correspondence, we further define the new angle

$$
\theta \equiv 2 \theta^{\prime} \quad \bmod 2 \pi
$$

so that three planes $\mathcal{P}_{i}(i=1,2,3)$ correspond, respectively, to $\theta=0,-2 \pi / 3$, and $2 \pi / 3$. Just after the breakdown of partial synchronization, the angle $\theta$ behaves as

(J1) $\theta \sim 0$ for $x^{(1)} \sim x^{(2)}$,

(J2) $\theta \sim-2 \pi / 3$ for $x^{(2)} \sim x^{(3)}$,

(J3) $\theta \sim 2 \pi / 3$ for $x^{(3)} \sim x^{(1)}$,

(J4) $\theta$ distributes on the plane $\mathcal{P}$ for no partial synchronization.

The elements $(\mathrm{J} 1-\mathrm{J} 4)$ correspond, respectively, to the aforementioned (V1-V4). The duration time of each of (J1)(J3) equally distributes according to the power law with the exponent $-3 / 2$ as shown in Fig. 6 . The time series of $r_{n}$ and $\theta_{n}$ are shown in Fig. 11. The former is totally chaotic. The element (J4) appears as a burst phase in $\theta_{n}$.

Switching of the pair of quasipartial synchronizations is described by rotation in the plane $\mathcal{P}$. We define the total rotation angle as

$$
\Theta_{n} \equiv \sum_{t=0}^{n-1}\left(\theta_{t+1}-\theta_{t}\right) .
$$

Note that $\Theta$ is defined not on $(-\pi, \pi]$ but on $(-\infty, \infty)$. The time series of $\Theta_{n}$ and the MSDs of $\Theta$ are shown in Figs. 12 and 13. Transition from anomalous subdiffusion $\left\langle X_{n}^{2}\right\rangle \propto \sqrt{n}$ to normal diffusion $\left\langle X_{n}^{2}\right\rangle \propto n$ appears. This behavior was observed in the on-off diffusion [28,29]. We will compare these numerical results with those from the theory by use of the CTRW jump model. The general framework of the CTRW model and its implementation to our model are, respectively, given in Appendices A and B. 


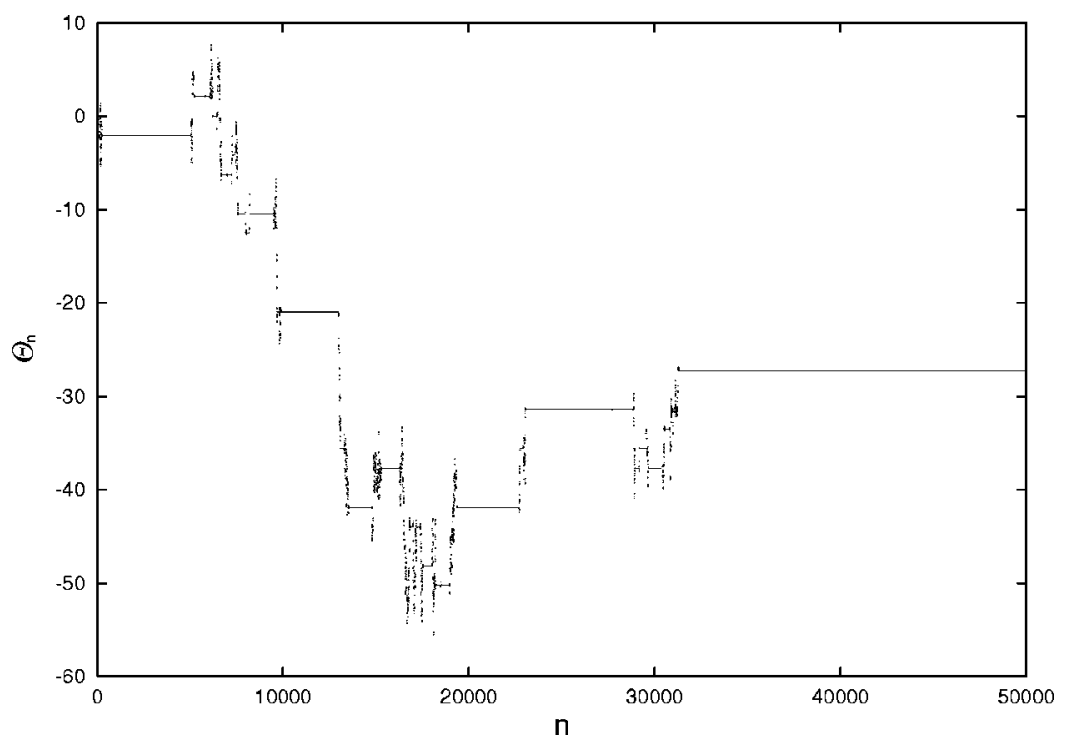

FIG. 12. Time series of $\Theta_{n}$.

\section{A. Statistical properties and scaling forms}

\section{Velocity model for $X$}

From the results of Appendix B, the MSD is given in the scaling form as

$$
\frac{\left\langle X_{t}^{2}\right\rangle}{2 D_{V} t}=\phi_{V}\left(\frac{t}{4 \tau}\right)
$$

where

$$
D_{V}=\frac{2 v^{2} \tau}{3}=\frac{2 v^{2} D_{\perp}}{3\left(\lambda_{\perp}^{(1)}\right)^{2}},
$$

and the scaling function is given by

$$
\phi_{V}(z)=z[1-\operatorname{erf}(\sqrt{z})]+\frac{e^{-z}}{2 \sqrt{\pi z}}(3-2 z)+\left(1-\frac{3}{4 z}\right) \operatorname{erf}(\sqrt{z}),
$$

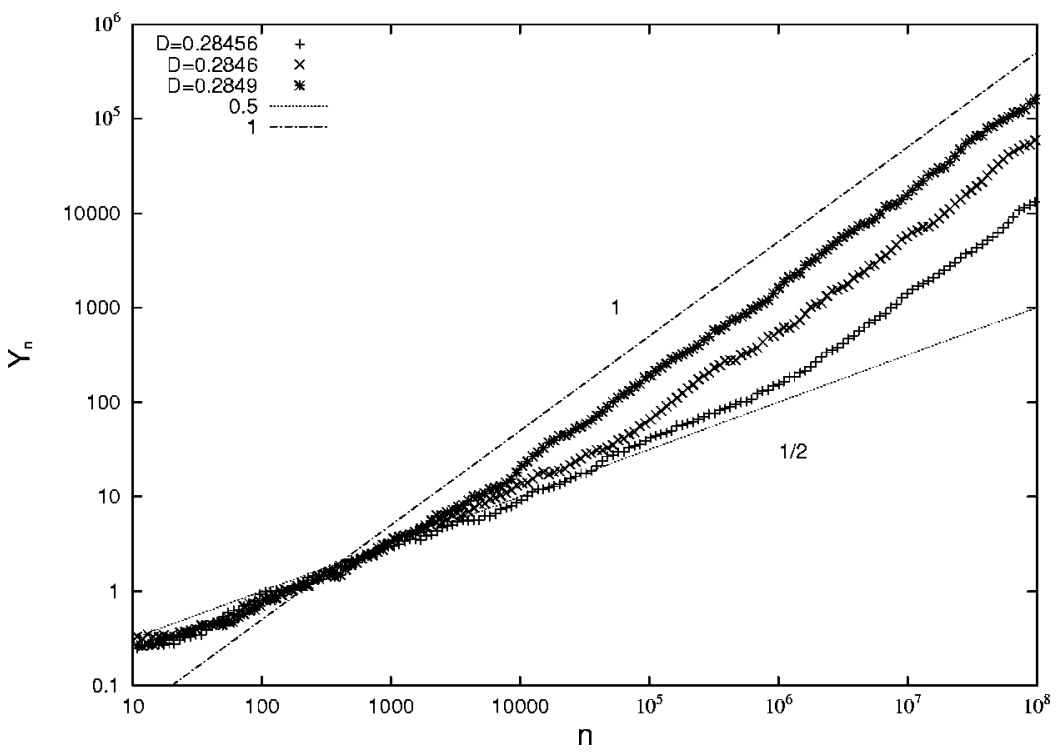

and $\tau$ denotes the characteristic time given explicitly by Eq. (B11), which separates the two kinds of diffusion. Note that $\phi_{V}(z) \sim 1$ for $z \gg 1$ and $\phi_{V}(z) \propto z$ for $z \ll 1$. Thus, the following transition from the ballistic motion to the normal diffusion is obtained

$$
\left\langle X_{n}^{2}\right\rangle \propto \begin{cases}n & (n \gg \tau) \\ n^{2} & \left(t^{*} \ll n \ll \tau\right),\end{cases}
$$

where $t^{*}=\alpha^{2} \tau$ is the characteristic time given by Eq. (B11) at which the ballistic motion starts.

\section{Jump model for $\Theta$}

From the results of Appendix B, the MSD is given in the scaling form as

$$
\frac{\left\langle\Theta_{t}^{2}\right\rangle}{2 D_{J} t}=\phi_{J}\left(\frac{t}{4 \tau}\right)
$$

FIG. 13. Mean square displacements $Y_{n}$ $=\left\langle\Theta_{n}^{2}\right\rangle$ for $D=0.28456,0.2846,0.2849$. Two kinds of the power laws Eq. (47) are given by lines. 


\section{SYNCHRONIZATION AND INTERMITTENCY IN THREE ...}

PHYSICAL REVIEW E 67, 016212 (2003)

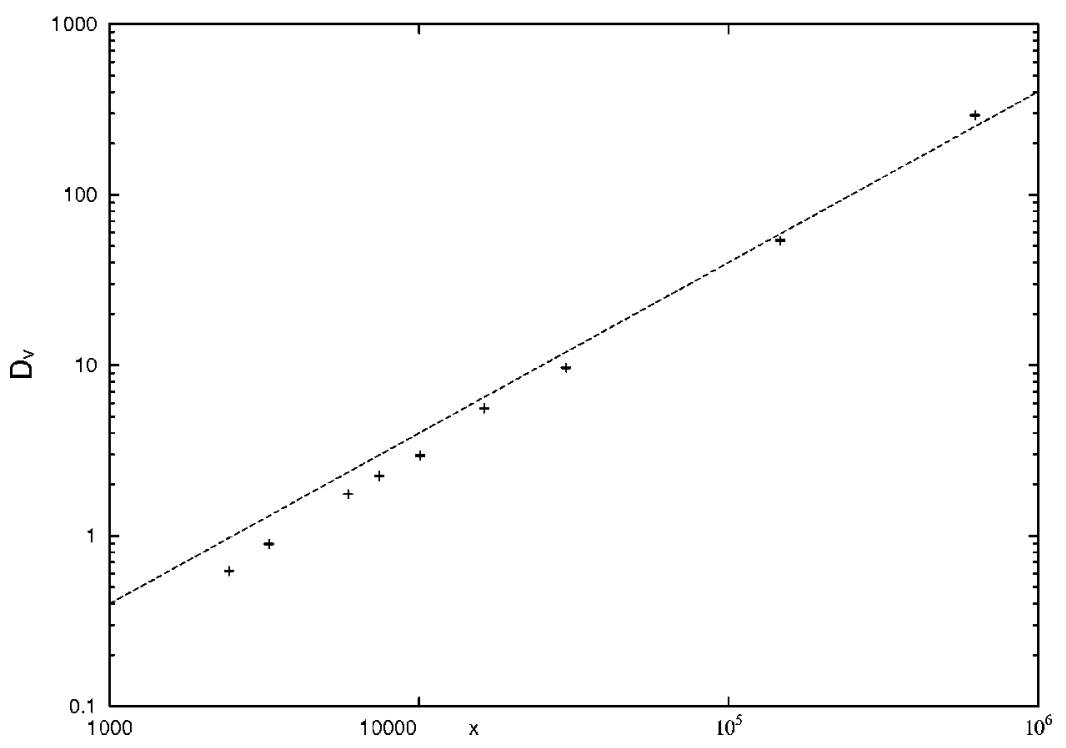

FIG. 14. Dependence on the transverse Lyapunov exponent $\lambda_{\perp}^{(1)}$ of the diffusion constant $D_{V}$, where $x=\left[\lambda_{\perp}^{(1)}\right]^{-2}$. Theoretical estimation Eq. (41) is given by the dashed line.
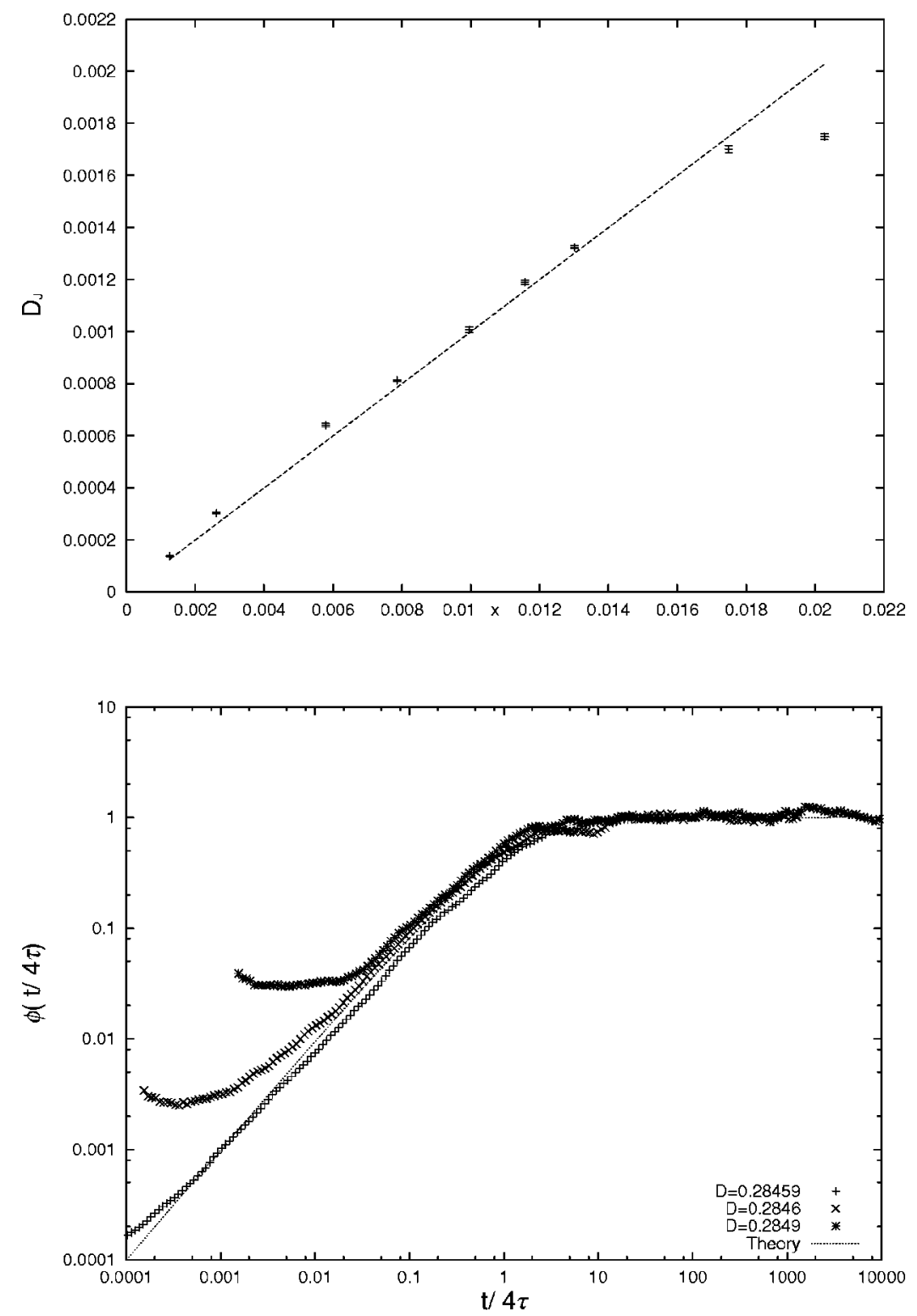

FIG. 15. Dependence on the transverse Lyapunov exponent $x=\lambda_{\perp}^{(1)}$ of the diffusion constant $D_{J}$. Theoretical estimation Eq. (45) is given by the dashed line.

FIG. 16. Scaling relations $\left\langle X_{t}^{2}\right\rangle / 2 D_{V} t$ vs $t / 4 \tau$ for $D=0.28459,0.2846,0.2849$. Theoretical estimation Eq. (42) is given by the dotted line. 


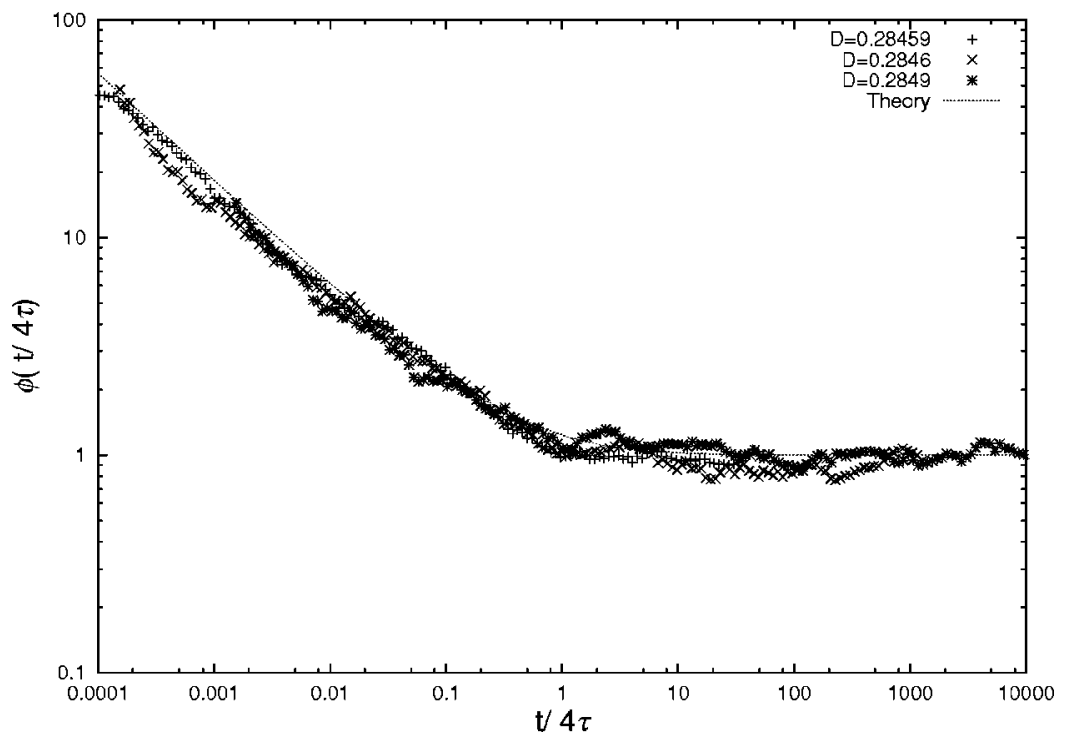

FIG. 17. Scaling relations $\left\langle\Theta_{t}^{2}\right\rangle / 2 D_{J} t$ vs $t / 4 \tau$ for $D=0.28459,0.2846,0.2849$. Theoretical estimation Eq. (46) is given by the dotted line. where

$$
D_{J}=\frac{\Sigma^{2}}{2 \bar{t}}=\frac{\Sigma^{2}}{c} \lambda_{\perp}^{(1)}
$$

and

$$
\phi_{J}(z)=\frac{1+\operatorname{erf}(\sqrt{z})}{2}+\frac{1}{2 \sqrt{\pi z}}\left(e^{-z}+\frac{\sqrt{\pi}}{2} \frac{\operatorname{erf}(\sqrt{z})}{\sqrt{z}}\right)
$$

and $\bar{t}$ and $\Sigma^{2}$ denote the average laminar duration given by Eq. (B9) and the second moment of the jump width given by Eq. (B1), respectively. The first and the second terms approach 1 and 0 , respectively, for $z \rightarrow \infty$, and $1 / 2$ and $1 / \sqrt{\pi z}$, respectively, for $z \rightarrow 0$, so that the following transition from anomalous subdiffusion to normal diffusion is observed

$$
\left\langle\Theta_{n}^{2}\right\rangle \propto \begin{cases}n & (n \gg \tau) \\ \sqrt{n} & \left(t^{*} \ll n \ll \tau\right) .\end{cases}
$$

This behavior was observed and analyzed [28,29] in the phenomenon called on-off diffusion [30].

\section{B. Comparison between the CTRW theory and numerical results}

We performed numerical simulations for the coupled logistic map to obtain the MSDs $\left\langle X_{n}^{2}\right\rangle$ and $\left\langle\Theta_{n}^{2}\right\rangle$. Each of the diffusion constants $D_{V}$ and $D_{J}$ is estimated by the least squares method for $t \gg \tau$, where $\tau$ denotes the characteristic time given explicitly by Eq. (B11) which separates the two kinds of diffusion.

Their dependences on the transverse Lyapunov exponent $\lambda_{\perp}^{(1)}$ are shown in Figs. 14 and 15. Our theory $D_{V}$ $\propto\left(\lambda_{\perp}^{(1)}\right)^{-2}$ shown in Eq. (41) and $D_{J} \propto \lambda_{\perp}^{(1)}$ shown in Eq. (45) are quantitatively in good agreement with numerical results. In Figs. 16 and 17, scaling relations $\left\langle X_{t}^{2}\right\rangle / 2 D_{V} t$ vs $t / 4 \tau$ and $\left\langle\Theta_{t}^{2}\right\rangle / 2 D_{J} t$ vs $t / 4 \tau$ are plotted and compared with our theo- retical curves $\phi_{V}$ and $\phi_{J}$, respectively. Good agreement is also found here.

\section{SUMMARY}

We studied the synchronization and the desynchronization problems in a coupled three-chaotic oscillator system to clarify their differences from those in a coupled twooscillator system. We discussed the synchronizationdesynchronization phenomenon with a simple model. For this purpose, we constructed a coupled map system using interacting kicked relaxators. First, we examined the complete and partial synchronization of the equivalently coupled three-chaotic map system, calculating their stability parameters. Furthermore, the statistical dynamics associated with the breakdown of the partial synchronization were studied by paying particular attention to the comparison of the statistics of on-off intermittency. Finally, we discussed two kinds of diffusion caused by breakdown of partial synchronization. Based on the CTRW theory, we analytically derived MSDs to find anomalous subdiffusion and ballistic motion followed by eventual normal diffusion. The statistical properties in various scaling forms were also derived. Good agreement between the theory and numerical results was also confirmed. We hope that the breakdown of partial synchronization and its statistical properties discussed in the present paper will be confirmed by experiment.

\section{ACKNOWLEDGMENTS}

We would like to thank Hiroki Hata for illuminating discussions.

\section{APPENDIX A: GENERAL FRAMEWORK OF CONTINUOUS-TIME RANDOM WALK}

Following the description of Zumofen and Klafter [31], we review the general framework of the CTRW theory. In the CTRW framework the random-walk process is entirely specified by $\psi(r, t)$, the probability density to move a dis- 
tance $r$ in time $t$ in a single motion. $\psi(r, t)$ can be either the decoupled case

$$
\psi(r, t)=\psi(t) \lambda(r),
$$

or the coupled case

$$
\psi(r, t)=p(r \mid t) \psi(t)
$$

where $p(r \mid t)$ is the conditional probability to move a distance $r$ in time $t$. The probability density $P(r, t)$ to be at location $r$ at time $t$ will be calculated in terms of $\psi(r, t)$ for the jump model and the velocity model. In order to obtain $P(r, t)$, we define the probabilities $\Psi(t)$ and $\Psi(r, t) . \Psi(t)$, needed for the jump model, is the probability for not leaving a position up to time $t$. This is related to $\psi(r, t)$ by

$$
\Psi(t)=\int_{t}^{\infty} d t^{\prime} \int d r \psi\left(r, t^{\prime}\right) .
$$

Similarly, for the velocity $\Psi(r, t)$ denotes the probability to pass at location $r$ at time $t$ in a single motion event; thus, we may write

$$
\Psi(r, t)=p(r \mid t) \int_{t}^{\infty} d t^{\prime} \int_{|r|}^{\infty} d r^{\prime} \psi\left(r^{\prime}, t^{\prime}\right) .
$$

In order to derive recursive expressions for $P(r, t)$, we consider $Q(r, t)$, the probability to arrive at $r$ exactly at time $t$ and to stop before randomly choosing a new direction. Irrespective of which model we choose, the following recursive relation holds:

$$
Q(r, t)=\int d r^{\prime} \int_{0}^{t} d t^{\prime} Q\left(r-r^{\prime}, t-t^{\prime}\right) \psi\left(r^{\prime}, t^{\prime}\right)+\delta(r) \delta(t) .
$$

The probability density $P_{J}(r, t)$ for the jump model is then related to $Q(r, t)$ in the following way:

$$
P_{J}(r, t)=\int_{0}^{t} d t^{\prime} Q\left(r, t-t^{\prime}\right) \Psi\left(t^{\prime}\right) .
$$

Analogously, for the velocity model we assume that the probability density $P_{V}(r, t)$ is the probability to stop or pass at location $r$ at time $t$ and, thus, we have

$$
P_{V}(r, t)=\int d r^{\prime} \int_{0}^{t} d t^{\prime} Q\left(r-r^{\prime}, t-t^{\prime}\right) \Psi\left(r^{\prime}, t^{\prime}\right) .
$$

In the Fourier $(r \rightarrow k)$ and Laplace $(t \rightarrow s)$ spaces, we have

$$
\begin{gathered}
P_{J}(r, t)=\frac{\Psi(s)}{1-\psi(k, s)}, \\
P_{V}(r, t)=\frac{\Psi(k, s)}{1-\psi(k, s)},
\end{gathered}
$$

where we introduce for the Fourier and/or Laplace transforms the convention that the arguments indicate in which space the function is defined, e.g., $P(k, s)$ is the FourierLaplace transform of $P(r, t)$. The corresponding MSDs are given in the Laplace space by

$$
\left\langle r^{2}\right\rangle_{J, V}(s)=-\left.\frac{d^{2} P_{J, V}(k, s)}{d k^{2}}\right|_{k=0} .
$$

\section{APPENDIX B: IMPLEMENTATION OF CTRW THEORY TO OUR MODEL}

For implementation of the CTRW model in our system, we must specify the waiting time distribution $\psi(t)$ and related function forms $\lambda(r)$ for the jump model and $p(r \mid t)$ for the velocity model.

As far as MSDs are concerned, a total specification of $\lambda(r)$ is not needed. We need only to specify its nonzero and finite second moment

$$
\Sigma^{2} \equiv \int_{-\infty}^{\infty} r^{2} \lambda(r) d r
$$

and the symmetry $\lambda(r)=\lambda(-r)$. The latter implies that no drift motion is considered in the jump model.

The coupled memory $p(r \mid t)$ for the velocity model is constructed along the elements $(\mathrm{V} 1-\mathrm{V} 3)$ as

$$
p(r \mid t)=\frac{1}{3}\{\delta(r)+\delta(r-v t)+\delta(r+v t)\},
$$

where the first, the second, and the third term describe the quiescent state (V1), the uniform motion with positive constant velocity $v(\mathrm{~V} 2)$, and with negative constant velocity $-v(\mathrm{~V} 3)$, respectively.

Thus, the key functions $\psi(r, t), \psi(k, s)$, and $\Psi(k, s)$ required in Appendix A are explicitly given by

$$
\begin{gathered}
\psi(r, t)=\frac{1}{3}\{\delta(r)+\delta(r-v t)+\delta(r+v t)\} \psi(t), \\
\psi(k, s)=\frac{1}{3}\{\psi(s)+\psi(s-i k v)+\psi(s+i k v)\}, \\
\Psi(k, s)=\frac{1}{3}\left\{\frac{1-\psi(s)}{s}+\frac{1-\psi(s-i k v)}{s-i k v}+\frac{1-\psi(s+i k v)}{s+i k v}\right\} .
\end{gathered}
$$

In order to specify the waiting time distribution $\psi(t)$, we aim to develop a picture in which, when the velocity is in its laminar phase of $\Delta^{1,2}$ or $\theta$, the test particle stays in the vicinity of one of the invariant manifolds (stays at a site of the random walk), until a burst velocity appears, which makes the test particle jump to the vicinity of a neighboring invariant manifold (jump to neighboring sites of the random walk) for the jump model. For the velocity model a burst velocity causes switching between the states $(\mathrm{V} 1-\mathrm{V} 3)$.

As observed numerically, the laminar time probability density satisfies a power law in a certain interval of time much smaller than the characteristic time of the system. In 
this section, the laminar time probability density is derived analytically by introducing a simple picture of dynamics in the laminar phase [28].

As shown for the on-off intermittency, the motion in the vicinity of the invariant manifolds on a logarithmic scale can be regarded as classical Brownian motion [32-34]. As the distance from the invariant manifolds becomes larger, nonlinearity causes reinjection to the manifolds, which appears as a burst phase in the time series of $\Delta^{1,2}$ or $\theta$.

Rewriting Eq. (29) in the continuous-time form, we have the linearized equation governing the dynamics of $r(t)$ in the laminar regime

$$
\frac{d r(t)}{d t}=\Lambda^{(1)}(t) r(t),
$$

where $\Lambda^{(1)}(t)$ is the local Lyapunov exponent. On the logarithmic scale, the dynamics in the laminar phase then obey

$$
\frac{d l(t)}{d t}=\Lambda^{(1)}(t),
$$

with $l(t) \equiv \log r(t)$ [35]. These dynamics exist until the nonlinearity becomes effective. We assume that the nonlinearity is no longer negligible at $l=l_{0}$.

Let $\tau_{1}, \tau_{2}, \ldots$ be the durations of the laminar phase, which can be approximated by the first passage time of the Wiener process Eq. (B7), in which the test particle starts from $l=l_{c}$ and arrives at $l=l_{0}$. We can also approximate the starting point as $l_{c}=\log \langle r(t)\rangle$ by use of the average distance from the invariant manifold $\langle r(t)\rangle$.

Thus, it becomes obvious that the mean drift velocity of the random variable $\log r(t)$ is given by the transverse Lyapunov exponent $\lambda_{\perp}^{(1)}$ in Eq. (30), whose variance is given by $2 D_{\perp}$. The probability density of the first passage time of the Wiener process Eq. (B7) with starting point $l=l_{c}$, goal $l=l_{0}>l_{c}$, positive drift velocity $\lambda_{\perp}^{(1)}$, and variance $2 D_{\perp}$ is given by [36]

$$
\psi(t)=\frac{c}{\sqrt{4 \pi D_{\perp}}} t^{-3 / 2} \exp \left[-\frac{\left(\lambda_{\perp}^{(1)} t-c\right)^{2}}{4 D_{\perp} t}\right],
$$

where $c=l_{0}-l_{c}>0$. We identify the above first passage time with the laminar phase duration as well as the waiting time of the CTRW theory below.

It is clear now that the power law of the laminar time distribution function is multiplied by an exponential function which guarantees the existence of the first moment. The average laminar duration is given by

$$
\bar{t}=\int_{0}^{\infty} t \psi(t) d t=\frac{c}{\lambda_{\perp}^{(1)}} .
$$

The Laplace transform of $\psi(t)$ is given by

$$
\psi(s)=\exp [\alpha(1-\sqrt{1+4 \tau s})],
$$

where $\tau$ and $\alpha$ are defined as

$$
\tau=\frac{D_{\perp}}{\left(\lambda_{\perp}^{(1)}\right)^{2}}, \quad \alpha=\frac{\lambda_{\perp}^{(1)} c}{2 D_{\perp}}=\frac{\bar{t}}{2 \tau},
$$

where the latter is the ratio of two characteristic time scales. The three independent parameters $\lambda_{\perp}^{(1)}, D_{\perp}$, and $c$ are contained in $\psi(t)$. However, $\psi(s)$ contains only two independent parameters. This originates from the scaling invariance of $\psi(t)$ and $\psi(s)$ under the transformation $c \rightarrow u c, \lambda_{\perp}^{(1)}$ $\rightarrow u \lambda_{\perp}^{(1)}$, and $D_{\perp} \rightarrow u^{2} D_{\perp}$ for an arbitrary real number $u$, so that the two parameters $\tau$ and $\alpha$ are relevant to on-off intermittency.

For $\tau s \ll 1$ or $1 \ll \tau s \ll 1 / \alpha^{2}$, we can approximate $\psi(s)$ as

$$
\psi(s) \approx 1+\alpha[1-\sqrt{1+4 \tau s}] .
$$

As long as $\alpha=\bar{t} /(2 \tau) \ll 1$, the above expression also holds for $\tau s \sim 1$, as asserted by the theorem of l'Hôpital in the limit $\alpha \rightarrow 0$. In many cases, $\tau \propto \epsilon^{-2}$ and $\bar{t} \propto \epsilon^{-1}$ are satisfied, where $\epsilon$ denotes the parameter distance from the bifurcation point, so that the relation $\alpha \ll 1$ is satisfied in the vicinity of the bifurcation point.

We estimate the MSDs $\left\langle X_{t}^{2}\right\rangle$ and $\left\langle\Theta_{t}^{2}\right\rangle$ by using the inverse Laplace transforms of $\left\langle r^{2}\right\rangle_{V}(s)$ and $\left\langle r^{2}\right\rangle_{J}(s)$ in Eq. (A10), respectively. Using the waiting time distribution Eq. (B12), we have

$$
\begin{gathered}
\left\langle X_{t}^{2}\right\rangle=\frac{4 v^{2}}{3} \mathcal{L}^{-1}\left[\frac{1}{2 s^{3}}-\frac{1}{2 s^{3} \sqrt{1+4 \tau s}}\right], \\
\left\langle\Theta_{t}^{2}\right\rangle=\frac{\Sigma^{2}}{4 \alpha \tau} \mathcal{L}^{-1}\left[\frac{1}{s^{2}}+\frac{\sqrt{1+4 \tau s}}{s^{2}}\right],
\end{gathered}
$$

where $\Sigma^{2}=\int_{-\infty}^{\infty} r^{2} \lambda(r) d r=\left.\left[d^{2} \lambda(k) / d(i k)^{2}\right]\right|_{k=0}$.
[1] A. Pikovsky, M. Rosenblum, and J. Kurths, Synchronization (Cambridge University Press, Cambridge, 2001).

[2] E. Mosekilde, Yu. Maistrenko, and D. Postnov, Chaotic Synchronization, Applications to Living Systems (World Scientific, Singapore, 2002).

[3] H. Fujisaka and T. Yamada, Prog. Theor. Phys. 69, 32 (1983).

[4] P. Berge, Y. Pomeau, and C. Vidal, Order within Chaos (Wiley, New York, 1986), Chap. IX.
[5] T. Yamada and H. Fujisaka, Prog. Theor. Phys. 70, 1240 (1983).

[6] H. Fujisaka and T. Yamada, Prog. Theor. Phys. 74, 918 (1985).

[7] H. Fujisaka and T. Yamada, Prog. Theor. Phys. 75, 1087 (1986).

[8] J. Redondo, D. Roldan, and G.J. de Valcarcel, Phys. Lett. A 210, 301 (1996).

[9] M. Sauer and F. Kaiser, Phys. Rev. E 54, 2468 (1996). 
[10] S.C. Venkataramani, T.M. Antonsen, Jr., E. Ott, and J.C. Sommerer, Physica D 96, 66 (1996).

[11] H. Tominaga, H. Fujisaka, and W. Just, J. Phys. Soc. Jpn. 66, 3406 (1997).

[12] E. Ott and J.C. Sommerer, Phys. Lett. A 188, 39 (1994).

[13] P.S. Landa, A.A. Zaikin, M.G. Rosenblum, and J. Kurths, Phys. Rev. E 56, 1465 (1997).

[14] H. Fujisaka, K. Ouchi, H. Hata, B. Masaoka, and S. Miyazaki, Physica D 114, 237 (1998).

[15] H. Hata and S. Miyazaki, Phys. Rev. E 55, 5311 (1997).

[16] S. Miyazaki and H. Hata, Phys. Rev. E 58, 7172 (1998).

[17] T. Yamada, K. Fukushima, and T. Yazaki, Prog. Theor. Phys. Suppl. 99, 120 (1989).

[18] P.W. Hammer, N. Platt, S.M. Hammel, J.F. Heagy, and B.D. Lee, Phys. Rev. Lett. 73, 1095 (1994).

[19] F. Rodelsperger, A. Cenys, and H. Benner, Phys. Rev. Lett. 75, 2594 (1996).

[20] Y.H. Yu, K. Kwak, and T.K. Li, Phys. Lett. A 198, 34 (1995).

[21] A. Cenys, A. Namajunas, A. Tamasevicius, and T. Schneider, Phys. Lett. A 213, 259 (1996).

[22] T. John, R. Stannarius, and U. Behn, Phys. Rev. Lett. 83, 749 (1999).

[23] H. Shirahama, K. Fukushima, N. Yoshida, and K. Taniguchi, IEEE Trans. CAS. 45, 1114 (1998).
[24] T. Yamada, K. Fukushima, and T. Yamazaki, Prog. Theor. Phys. Suppl. 99, 120 (1989).

[25] H. Tsuruda, H. Shirahama, K. Fukushima, M. Nagadome, and M. Inoue, Chaos 11, 410 (2001).

[26] L.M. Pecora and T.L. Carroll, Phys. Rev. Lett. 64, 821 (1990); Phys. Rev. A 44, 2374 (1991).

[27] A.V. Taborov and Yu.L. Maistrenko, and E. Mosekilde, Int. J. Bifurcation Chaos Appl. Sci. Eng. 10, 1051 (2000).

[28] S. Miyazaki, J. Phys. Soc. Jpn. 69, 2719 (2000).

[29] S. Miyazaki, T. Harada, and A. Budiyono, Prog. Theor. Phys. 106, 1051 (2001).

[30] T. Harada, H. Hata, and H. Fujisaka, J. Phys. A 32, 1557 (1999).

[31] G. Zumofen and J. Klafter, Phys. Rev. E 47, 851 (1993).

[32] A. Cenys and H. Lustfeld, J. Phys. A 29, 11 (1996).

[33] A. Cenys, A.N. Anagnostopoulos, and G.L. Bleris, Phys. Lett. A 224, 346 (1997).

[34] A. Cenys, A.N. Anagnostopoulos, and G.L. Bleris, Phys. Rev. E 56, 2592 (1997).

[35] T. Yamada and H. Fujisaka, Prog. Theor. Phys. 76, 582 (1986).

[36] L.M. Ricciardi, Diffusion Processes and Related Topics in Biology, Lecture Notes in Biomathematics Vol. 14 (Springer, Berlin, 1977), p. 504. 